\title{
COVID-19: The Potential Difference in High Resolution Computed Tomography Findings between Vaccinated and Unvaccinated Cases among The Adult Egyptian Population
}

\author{
Dena M Serag, Eman R Selima
}

Department of Radiodiagnosis, Faculty of Medicine, Menoufia University, Egypt

Corresponding author: Dena M Serag, Phone number: (01000205159), E-mail :denaserag@yahoo.com

\begin{abstract}
Background: As the world experiences successive waves of corona virus disease (COVID-19) pandemic, global efforts to develop and distribute an effective vaccine produced several promising options. The aim of our study was to assess the difference in pulmonary involvement between vaccinated and unvaccinated patients among Egyptians.

Patients and methods: Our study involved 234 patients who were tested positive for COVID-19 and had classic symptoms. They were split into 2 groups: group 1 (131 unvaccinated patients), and group 2 (103 fully vaccinated patients). Patients underwent high resolution computed tomography (HRCT) of the chest. All scans were evaluated, and CT severity score (CT-SS) was calculated.

Results: For group 1 the patients' ages ranged from 19 to 84, mean $=44.5$ years $( \pm 16.2 \mathrm{SD}), 76(58 \%)$ males and $55(42 \%)$ females, while group 2 age ranged from 18 to 85 , mean $( \pm \mathrm{SD})=42.2$ years $( \pm 16.6), 59(57.3 \%)$ males and $44(42.7 \%)$ females". The presence of ground glass opacities and white lung sign varied significantly between both groups ( $\mathrm{P}=0.001$ and 0.002 respectively). For other recorded signs no significant difference was detected. The mean computed tomography severity score (CT-SS) for group 1 was 11.25 $( \pm 6.6 \mathrm{SD})$. While for group 2 it was $6.32( \pm 4.33 \mathrm{SD})$. The difference between studied groups was highly significant $(\mathrm{P}<0.001)$.

Conclusion: Our results showed that COVID vaccine could effectively reduce the extent and severity of lung involvement in vaccinated patients compared with unvaccinated patients. Further wide-ranging clinical studies are needed to support our results.
\end{abstract}

Keywords: Computed tomography, Coronavirus, COVID-19, Vaccine.

\section{BACKGROUND}

Coronaviruses consist of a large group of viruses which often lead to upper respiratory mild to moderate illnesses, as common cold. Unfortunately, the multiple new coronaviruses that developed from animals during the last twenty years caused severe critical health problems ${ }^{[1]}$.

In December 2019, a newly identified strain appeared leading to a pandemic that began in China. The incriminated virus is known as severe acute respiratory syndrome coronavirus 2 (SARS-CoV-2). The resulting morbidity is known as (COVID-19) disease ${ }^{[2]}$.

This current pandemic has caused a violent respiratory affection in more than one hundred and sixty million people worldwide and caused more than $2 \%$ deaths ${ }^{[3]}$. Since the commencement of the outbreak, the suspected or the confirmed cases underwent HRCT of the chest to assess the degree of pulmonary involvement ${ }^{[4]}$. On HRCT, ground glass opacities are the commonest pattern in COVID-19 pneumonia which means increased attenuation of the lung parenchyma without obscuring the vasculature and the bronchial markings. Bilateral sub-pleural lesions with lower lobar predominance are still the most popular pattern in many of the scanned cases ${ }^{[5]}$.

Reaching a rightful vaccine being effective and offering safety is crucial to abort COVID-19 outbreak. Continuous efforts are made to provide and develop many kinds of vaccines. For this foreseeable future, the populations must continue keeping their masks, washing their hands, maintain proper ventilation, social distancing, and stay away from crowds as possible ${ }^{[6]}$.

The aim of this work was to assess the difference in severity of lung involvement between vaccinated and unvaccinated patients.

\section{PATIENTS AND METHODS Cases:}

The current prospective case-control study was carried out in the period from $15^{\text {th }}$ April 2021 to $15^{\text {th }}$ July 2021 with enrollment of 234 cases.

Inclusion criteria: All enrolled participants had positive reverse transcription polymerase chain reaction (RT-PCR) or rapid test. All cases underwent HRCT chest during the $5^{\text {th }}$ to the $10^{\text {th }}$ day from the onset of the symptoms. The cases were split into two groups; group 1 unvaccinated patients and group 2 fully vaccinated patients (who had their second dose more than two weeks before developing symptoms). Most of group 2 participants were of medical personnel.

Exclusion criteria: the vaccinated cases who had confirmed previous infection or confirmed symptoms since the commencement of the outbreak. Cases less than 18 years old.

\section{Ethical approval:}

The study was approved by the Research Ethical Committee of Menoufia Faculty of Medicine. An informed consent was taken from every patient in this research. Every patient got an explanation for the purpose of the study.

All given data were used for the current medical research only. This work was performed in accordance with The Code of Ethics of the World Medical Association (Declaration of Helsinki) for studies involving humans.

\section{Image acquisition and analysis:}

Chest HRCT was performed on a 160- MDCT scanner (Aquilion Prime, Canon medical systems, Japan). Patients were examined in supine position, headfirst. Using single breath hold whenever possible and tolerable.

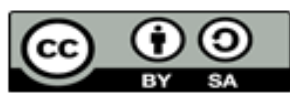

This article is an open access article distributed under the terms and conditions of the Creative Commons Attribution (CC BY-SA) license (http://creativecommons.org/licenses/by/4.0/) 
Image acquisition parameters: 100 kilo-voltage (KV), 250 milliampere/second (mAs), field of view $(\mathrm{FOV})=460 \mathrm{x}$ $460 \mathrm{~mm}$, slice thickness and inter-slice gap $1 \mathrm{~mm}$. Scans were reviewed on VITREA workstation, Japan for careful assessment.

All scans were evaluated by two expert radiologists with more than twelve years' experience. The check list consisted of:

1- The lesions' laterality either unilateral or bilateral.

2- The affected lobes of the lungs whether uni-lobar, bilobar, or multi-lobar.

3- Lesions' distribution either peripheral affecting the outer $1 / 3$ or central affecting the inner $2 / 3$.

4- Pattern of the lesions varying from ground glass opacity, consolidation, crazy paving appearance, and septal lines.

5- Presence of special signs as (vascular thickening, reverse halo, air bronchogram, or white lung sign).

6- Presence of extra-pulmonary imaging findings like pleural effusion or mediastinal lymph nodes.

7- Calculation of CT-severity score (CT-SS) (range from 0 : 25); anatomically, there are five lobes in both lungs. Every lung lobe was evaluated for the percentage of involvement in concordance with table $1^{[7-10]}$.

Table 1: The individual scores of lung lobes based on the degree of the affection ${ }^{[7-10]}$

\begin{tabular}{||l|l||}
\hline \hline Lobar involvement & Score \\
\hline No affection & $\mathbf{0}$ \\
\hline$<5 \%$ & $\mathbf{1}$ \\
\hline 5 to $<25 \%$ & $\mathbf{2}$ \\
\hline 25 to $<50 \%$ & $\mathbf{3}$ \\
\hline 50 to $<75 \%$ & $\mathbf{4}$ \\
\hline$\geq 75 \%$ & $\mathbf{5}$ \\
\hline \hline
\end{tabular}

8- According to CT-SS lung involvement was categorized into mild, moderate and severe involvement according to table $2^{[11]}$.

Table 2: Sum of scores of individual lobes giving the overall degree of affection of all lobes ${ }^{[11]}$.

\begin{tabular}{||l|l||}
\hline Total score & Severity \\
\hline 7 or less & Mild \\
\hline From 8 to1 7 & Moderate \\
\hline 18 or more & Severe \\
\hline \hline
\end{tabular}

\section{Statistical analysis}

Data were analyzed using an IBM compatible personal computer with SPSS statistical package version 23 (SPSS Inc. Released 2015. IBM SPSS statistics for windows, version 23.0, Armnok, NY: IBM Corp.). Categorical variables were presented as number (No) and percentage (\%), while quantitative variables were presented as mean and standard deviation (SD). Mann Whitney's test was performed for nonnormally distributed variables.

Chi-square test $\left(\boldsymbol{\chi}^{2}\right)$ (with $\mathrm{Z}$ test was used to compare column) was used to detect association between qualitative variables.

Whenever any of the studied cells were less than five, Fischer's exact test was applied. P-value of $<0.05$ was considered to be statistically significant.

\section{RESULTS}

Our study included 234 patients. They were divided into 2 age and gender matched groups.

The unvaccinated group (group 1) included 131 patients (76 $(58 \%)$ males \& $55(42 \%)$ females $)$, mean age $( \pm S D)=44.5$ years $( \pm 16.2)$, their ages ranged from 19 to 84 years (as shown in fig. 1, 3 \& 4). The vaccinated group (group 2) included 103 patients $(59(57.3 \%)$ males \& $44(42.7 \%)$ females $)$, mean age $=$ 42.2 years $( \pm 16.6 \mathrm{SD})$. Their ages ranged from 18 to 85 years (fig. 2).

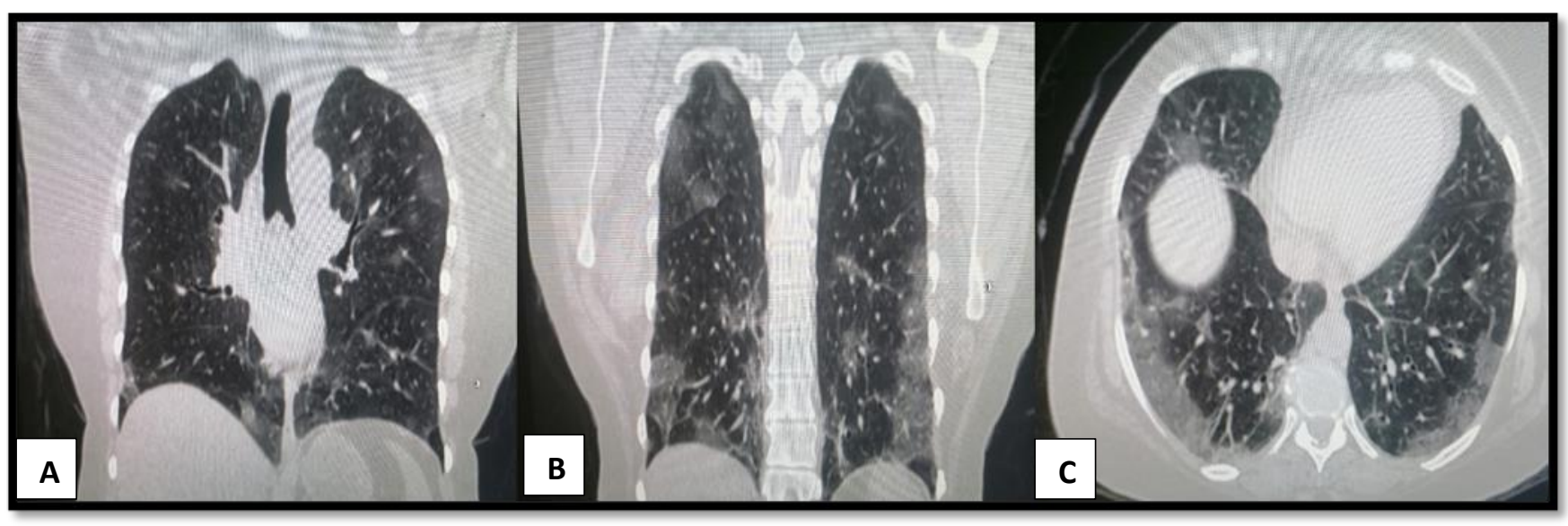

Fig. (1): Unvaccinated female patient scanned on the $5^{\text {th }}$ day of the onset of symptoms. A, B coronal and C axial MDCT scans (lung window) showing bilateral peripheral patchy ground glass opacities involving all lobes, mainly both lower lobes. CT-SS [15/25] 


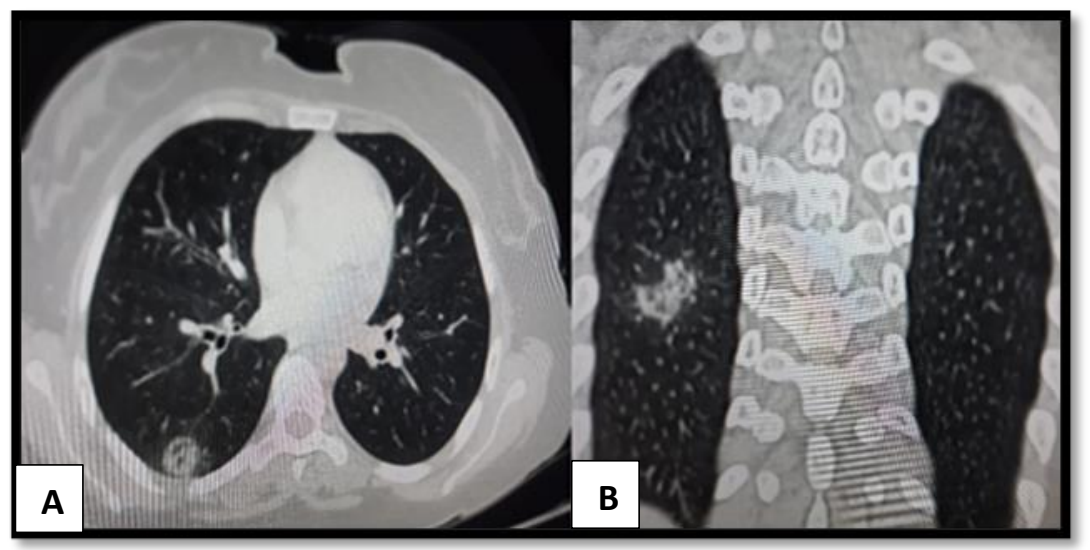

Fig. (2): Vaccinated female patient scanned on the $8^{\text {th }}$ day of the onset of the symptoms. A axial and B coronal thin section CT scans of the lung showing a single small right lower lobe patch of ground glass opacity with clear other lung lobes. CT-SS [1/25].

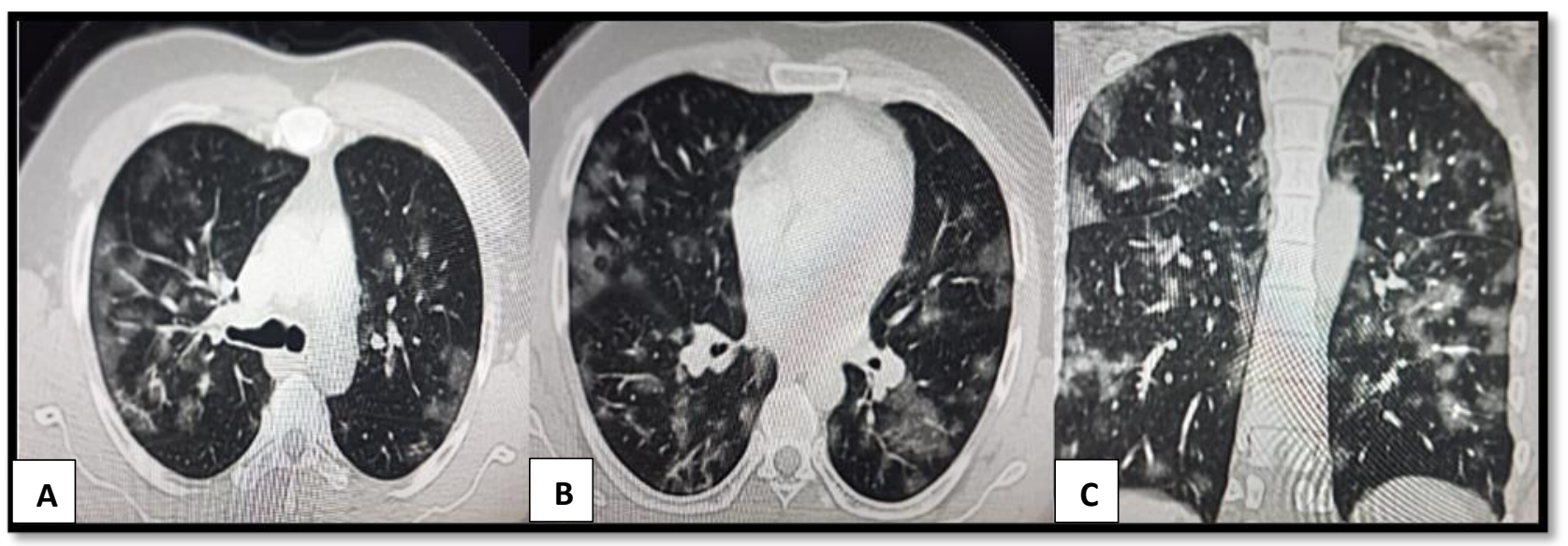

Fig. (3): Unvaccinated male patient scanned on the $7^{\text {th }}$ day of the onset of symptoms. A, B axial and C coronal CT scans of the lung showing extensive bilateral peripheral and central patchy ground glass opacities involving all lung lobes. CT-SS [17/25].

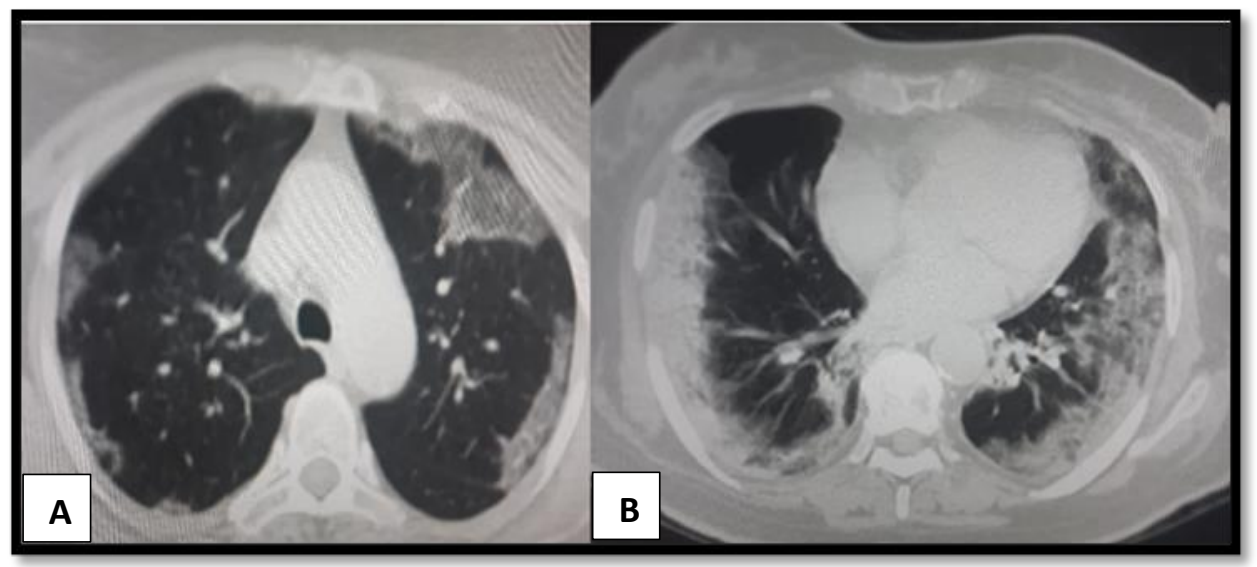

Fig. (4): Unvaccinated female patient scanned on the $8^{\text {th }}$ day of onset of symptoms. A and B axial CT scans of upper and lower lung lobes showing bilateral peripheral ground glass opacities and consolidation patches, with thickened interlobular lines (crazy paving pattern). CT-SS [14/25].

Patients' demographics are summarized in table 3. 
Table 3: Age and gender distribution in the studied groups

\begin{tabular}{||l|c|c|c||}
\hline & Group 1 $(\mathbf{n = 1 3 1})$ & Group 2 $(\mathbf{n = 1 0 3})$ & P value \\
\hline$\bullet \quad$ Age (mean \pm SD) & $44.54 \pm 16.22$ & $42.41 \pm 16.6$ & 0.255 \\
\hline Gender No. (\%) & $76(58.0)$ & $59(57.3)$ & 0.910 \\
$\bullet \quad$ Male & $55(42.0)$ & $44(42.7)$ & \\
\hline
\end{tabular}

Table 4 shows the different CT findings and distribution of lung involvement in both groups. The presence of ground glass opacities was significantly lower in group 2 (vaccinated) than in group 1 (unvaccinated). White lung was observed in 12 patients (9\%) in group 1 but none in group 2, and the difference was statistically significant.

Table 4: CT findings in both groups

\begin{tabular}{|c|c|c|c|}
\hline & $\begin{array}{l}\text { Group } 1(n=131) \\
\text { No. }(\%)\end{array}$ & $\begin{array}{l}\text { Group } 2(n=103) \\
\text { No. }(\%)\end{array}$ & $\underline{P \text { value }}$ \\
\hline \multicolumn{4}{|l|}{ Laterality } \\
\hline - No affection & $9(6.9)$ & $22(21.4)$ & 0.002 \\
\hline - Unilateral & $28(21.4)$ & $15(14.5)$ & \\
\hline - Bilateral & $94(71.7)$ & $66(64.1)$ & \\
\hline \multicolumn{4}{|l|}{ location } \\
\hline - $\quad$ Peripheral & $75(57.3)$ & $48(46.5)$ & 0.115 \\
\hline - Central & $5(3.8)$ & $4(3.9)$ & 1.00 \\
\hline - Both & $42(32)$ & $29(28.2)$ & 0.397 \\
\hline \multicolumn{4}{|l|}{ Lobes affected } \\
\hline - Uni-lobar & $20(15.3)$ & $11(10.6)$ & 0.304 \\
\hline - Bi-lobar & $52(39.6)$ & $36(35.0)$ & 0.457 \\
\hline - Multi-lobar & $50(38.2)$ & $34(33.0)$ & 0.414 \\
\hline \multicolumn{4}{|l|}{ Pattern } \\
\hline $\begin{array}{l}\bullet \quad \mathrm{GGO} \\
\end{array}$ & $121(92.4)$ & $80(77.7)$ & 0.001 \\
\hline - Consolidation & $47(35.9)$ & $34(33.0)$ & 0.647 \\
\hline \multicolumn{4}{|l|}{ Special signs } \\
\hline - $\quad$ Septal lines & $22(16.8)$ & $17(16.5)$ & 0.953 \\
\hline - Crazy paving & $31(23.7)$ & $22(21.4)$ & 0.676 \\
\hline - Air bronchogram & $46(35.1)$ & $34(33.0)$ & 0.736 \\
\hline - Reversed halo & $9(6.9)$ & $5(4.9)$ & 0.519 \\
\hline - Vascular prominence & $14(10.7)$ & $14(13.6)$ & 0.497 \\
\hline - White lung & $12(9.2)$ & $0(0.0)$ & 0.002 \\
\hline \multicolumn{4}{|l|}{ Extra pulmonary findings } \\
\hline - Lymphadenopathy & $18(13.7)$ & $15(14.6)$ & 0.858 \\
\hline - $\quad$ Pleural effusion & $22(16.7)$ & $14(13.6)$ & 0.864 \\
\hline
\end{tabular}

GGO: ground glass opacities

CT severity score for group 1 ranged from $0: 25$ with a mean value of $11.25( \pm 6.6 \mathrm{SD})$. while for group 2 the score ranged from $0: 14$ with a mean of $6.32( \pm 4.33 \mathrm{SD})$. There was highly significant difference between both groups $(\mathrm{P}<0.001) .($ table5)

Table 5: CT severity score in the studied groups

\begin{tabular}{|c|c|c|c|}
\hline & $\begin{array}{l}\text { Group } 1(n=131) \\
\text { No. }(\%)\end{array}$ & $\begin{array}{l}\text { Group } 2(n=103) \\
\text { No. }(\%)\end{array}$ & $P$ value \\
\hline CT-SS (mean \pm SD) & $11.25( \pm 6.60)$ & $6.32( \pm 4.33)$ & $<0.001$ \\
\hline \multicolumn{4}{|l|}{ CT-SS categories: } \\
\hline - $\quad$ Not affected & $9(6.9)$ & $22(21.4)^{*}$ & $<0.002$ \\
\hline - $\quad$ Mild & $43(32.8)$ & $42(40.8)$ & 0.621 \\
\hline - $\quad$ Moderate & $53(40.5)$ & $39(37.9)$ & 0.542 \\
\hline - $\quad$ Severe & $26(19.8)^{*}$ & $0(0.0)$ & $<0.001$ \\
\hline
\end{tabular}

* = statistically significant difference.

According to the CT-SS; cases were categorized as mild, moderate, and severe involvement as shown in figure $\mathbf{5}$ and table $\mathbf{5}$. 


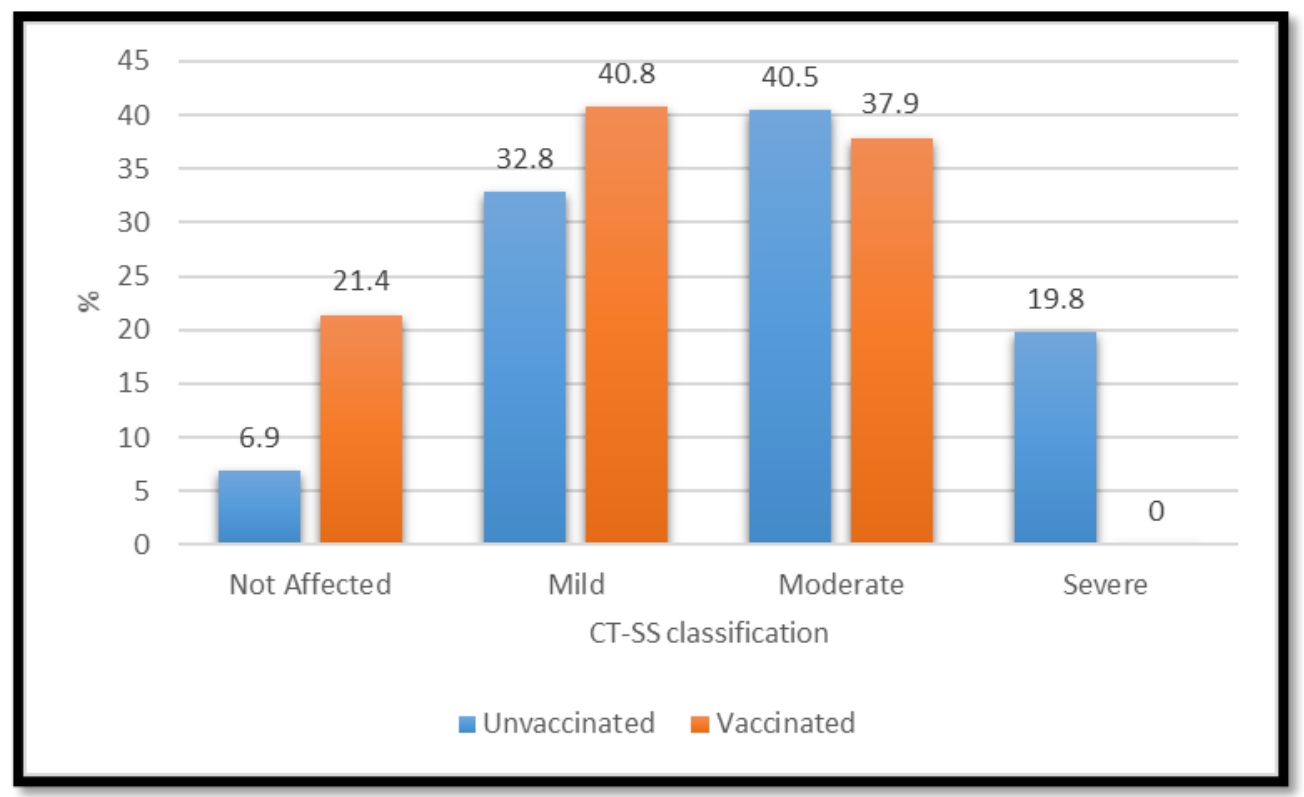

Fig. (5): The difference in CT-SS between vaccinated and unvaccinated patients.

\section{DISCUSSION}

The COVID-19 pandemic resulted in a dramatic unparalleled challenge to public health in addition to the devastating economic losses and social disruption. In order to end this pandemic, a large part of the world population needs to acquire immunity against the virus. Recent reports showed that $45.7 \%$ of the world population got at least one dose of the vaccine, 6.34 billion doses were administered worldwide, and that $11 \%$ of the Egyptian population have been partially or fully immunized till Sep $30^{\text {th }} 2021^{[12]}$. This study aimed to delineate the difference in extent of pulmonary involvement with COVID-19 manifestations between the vaccinated and the unvaccinated Egyptian patients.

In the current study 234 patients who tested positive COVID-19 by RT-PCR were separated into two groups: group 1 (131 unvaccinated patients), and group 2 (103 fully vaccinated patients). They were age and gender matched.

In our study CT-SS for group 1 ranged from $0: 25$ with a mean value of $11.25( \pm 6.6 \mathrm{SD})$. While for group 2 the score ranged from $0: 14$ with a mean of $6.32( \pm 4.33 \mathrm{SD})$ and the difference was highly significant. Our findings came in concordance with the results obtained by Madhu et al. ${ }^{[5]}$. They reported a mean CT-SS for unvaccinated patients $=14.7( \pm 7.3$ $\mathrm{SD})$ ranging from 3 to 21 . While for the vaccinated group the mean CT-SS was $5.3( \pm 2.9 \mathrm{SD})$ ranging from 3 to $8(\mathrm{P}<0.05)$.

Madhu et al. ${ }^{[5]}$ reported lung involvement in $88 \%$ of the unvaccinated group and $12 \%$ in the vaccinated group. This was quite different from our results. We recorded $93 \%$ lung involvement in unvaccinated group, and $78.6 \%$ in vaccinated group. This might be attributed to large variation in sample size (131 unvaccinated and 103 vaccinated in our study Vs 180 unvaccinated and 26 vaccinated in their study). Most of the affected patients in unvaccinated group in their study showed moderate and severe involvement $(43.7 \%$ and $25 \%$ respectively). For the vaccinated group mild involvement was more likely. This was consistent with our results. Most of the unvaccinated patients in our study had moderate to severe involvement (40.5\% and $20 \%$ respectively), while in the vaccinated group most patients (40.8\%) had mild involvement and none of the patients had severe involvement. Lakhia et al. ${ }^{[13]}$ also reported similar findings with significant difference in CT-SS between the fully vaccinated, the partially vaccinated and the unvaccinated patients $(\mathrm{P}<0.01)$ and mean CT-SS 3.5, 6.4 and 10 respectively.

\section{Limitation of the study:}

At time of conduction of the current study two vaccines were authorized and available for use in Egypt (Oxford AstraZeneca and Sinopharm), but we couldn't study the difference between them regarding the degree of improving lung involvement they provide. Also there were no sufficient data about the clinical outcome in both patient groups. Finally till the time this article was written, a limited number of studies with the same rationale were available for comparison.

\section{CONCLUSION}

Our results showed that COVID vaccine could effectively reduce the extent and severity of lung involvement in vaccinated patients compared with unvaccinated patients. Further wide-ranging and multi-center studies are needed to support our results.

\section{RECOMMENDATIONS}

Continuous implementation of mass vaccination campaigns is mandatory as it could reduce the severity of lung involvement with COVID-19 manifestations. Paying more attention and sustained efforts to third world countries as reports state that only $2.3 \%$ of population in developing countries have received the vaccine.

Financial support and sponsorship: None. Conflict of interest: None. 


\section{REFERENCES}

1. Coronaviruses | NIH (2020): National Institute of Allergy and Infectious Diseases. In: NIH Natl. Institue Allergy Infect. Dis. NIAID. https://www.niaid.nih.gov/diseasesconditions/coronaviruses.

2. Salehi S, Abedi A, Balakrishnan S et al. (2020): Coronavirus disease 2019 (COVID-19): A systematic review of imaging findings in 919 patients. American Journal of Roentgenology; 215(1): 87-93.

3. Roser M, Ritchie H, Ortiz-Ospina E et al. (2020): Coronavirus Pandemic (COVID-19). Our world in data. https://ourworldindata.org/coronavirus.

4. Xie X, Zhong Z, Zhao W et al. (2020): Chest CT for typical coronavirus disease 2019 (COVID-19) pneumonia: relationship to negative RT-PCR testing. Radiology,296(2): 41-45.

5. Madhu P, Santhosh D, Madhala K (2021): Comparison study of lung involvement in vaccinated and un vaccinated Covid patients. International Journal of Health and Clinical Research,4(10):229-233.

6. Marineci C, Văleanu A, Negreş S et al. (2021): COVID-19 vaccines. Farmacist.Ro.,1(198): 1155-1162.

7. Chang Y, Yu C, Chang S et al. (2005): Pulmonary sequelae in convalescent patients after severe acute respiratory syndrome: evaluation with thin-section CT. Radiology,236(3): 1067-1075.

8. Zhou S, Wang Y, Zhu T et al. (2020): CT features of coronavirus disease 2019 (COVID-19) pneumonia in 62 patients in Wuhan, China. American Journal of Roentgenology, 214(6): 1287-1294.

9. Pan F, Ye T, Sun P et al. (2020): Time course of lung changes at chest CT during recovery from coronavirus disease 2019 (COVID-19). Radiology, 295(3):715-721.

10. Ragab E, Mahrous A, El Sheikh G (2020): COVID-19 infection: epidemiological, clinical, and radiological expression among adult population. Egypt J Radiol Nucl Med., 51:236.

11.Saeed G, Gaba W, Shah A et al. (2021): Correlation between chest CT severity scores and the clinical parameters of adult patients with COVID-19 Pneumonia. Radiology Research and Practice,265(55):564-569.

12. Hannah R, Edouard M, Lucas R et al. (2020): Coronavirus Pandemic https://ourworldindata.org/coronavirus.

(COVID-19).

13. Lakhia T, Trivedi R (2021): The CT scan lung severity score and vaccination status in COVID-19 patients during the second wave in India: Perspective of an independent radiology https://doi.org/10.1101/2021.07.15.21260597. 